- Original Article

\title{
Factors Associated with Dry Eye Symptoms in Elderly Koreans: The Fifth Korea National Health and Nutrition Examination Survey 2010-2012
}

\author{
Kyong In Kim, Yong Soon Park*, Ryoung Hee Kim, Jeong Hyeon Kim \\ Department of Family Medicine, Hallym University Chuncheon Sacred Heart Hospital, Chuncheon, Korea
}

\begin{abstract}
Background: Dry eye disease is an aging-related ophthalmic disease that not only affects the daily activities but also causes deterioration in the quality of life. This study aimed to evaluate the factors associated with dry eye symptoms in elderly Koreans.

Methods: We investigated 4,185 subjects (men=1,787 and women=2,398) aged $\geq 65$ years from the fifth Korea National Health and Nutrition Examination Survey 2010-2012. Data were analyzed using multiple logistic regressions to identify the relationships between dry eye symptoms and other factors.

Results: The prevalence of dry eye symptoms was $17.9 \%$. After adjustment for confounding factors, dry eye symptoms were significantly associated with female sex (adjusted odds ratio [aOR], 1.806; 95\% confidence interval [CI], 1.410-2.313), a history of cataract (aOR, 1.683; 95\% CI, 1.255-2.255), suicidal ideation (aOR, 1.414; 95\% CI, 1.070-1.870), hypercholesterolemia (aOR, 1.289; 95\% CI, 1.025-1.621), age $\geq 80$ years (aOR, 0.538; 95\% CI, $0.337-0.859$ ), and sleep duration $\geq 9 \mathrm{~h} / \mathrm{d}$ (aOR, $0.524 ; 95 \% \mathrm{CI}, 0.330-0.834)$.

Conclusion: Among elderly Koreans, female sex, a history of cataract, suicidal ideation, and hypercholesterolemia may be the risk factors for dry eye symptoms, whereas sleep duration $\geq 9 \mathrm{~h} / \mathrm{d}$ can be a protective factor against dry eye symptoms.
\end{abstract}

Keywords: Dry Eye Syndromes; Risk Factors; Aged; Korea National Health and Nutrition Examination Survey

Received: May 11, 2017, Revised: August 12, 2017, Accepted: August 29, 2017

*Corresponding Author: Yong Soon Park https://orcid.org/0000-0002-8926-9836

Tel: +82-33-240-5311, Fax: +82-33-240-5440, E-mail: pyongs@hanmail.net 


\section{INTRODUCTION}

Aging in the South Korean population is progressing at a much faster pace than in populations in other developed countries. The elderly population was $7.2 \%$ in 2000 and $12.8 \%$ in 2015 , and the elderly population is expected to increase to $20 \%$ by 2026 and to $40 \%$ by $2058 .{ }^{1)} \mathrm{Ag}$ ing is accompanied by a decrease in mental and physical functions, particularly in the functions of the sensory organs, which affect daily life and health. Aging-related ophthalmic diseases, such as age-related macular degeneration, diabetic ophthalmopathy, cataract, glaucoma, dry eye disease, and low vision, have been increasing in incidence; these cause major economic loss to individuals and society and reduce the quality of life in the elderly. ${ }^{2)}$

Dry eye is a multifactorial disease affecting the tears and ocular surface and is accompanied by increased osmolarity of the tear film and inflammation of the ocular surface, which cause ocular discomfort, visual disturbance, and tear film instability, with potential damage to the ocular surface. ${ }^{3)}$ Studies on dry eye symptoms conducted since 2006 have shown prevalence rates ranging from $12.3 \%$ to $73.5 \%$ worldwide. ${ }^{4,5)}$ Surveys in South Korea have reported prevalence rates ranging from $8.0 \%$ to $30.3 \% .{ }^{6,7)}$ Because of the rapid increase in the elderly population, changes in the atmosphere, and modifications to the lifestyle, the prevalence of dry eye disease has been increasing, particularly in the elderly. Dry eye affects daily activities, such as reading, computer usage, television viewing, professional work, and driving, and can result in decreased visual acuity because of corneal turbidity and scarring. ${ }^{8)}$ These effects of dry eye have an enormous impact on the quality of life in the elderly.

Dry eye disease is a serious public health issue in elderly Koreans. Although various studies on dry eye disease have been conducted using the Korea National Health and Nutrition Examination Survey (KNHANES) in South Korea, most studies have been performed in the general population aged $\geq 19$ years old, and no study on the elderly population has been reported. Furthermore, all studies on dry eye disease in the elderly Korean population have been conducted among the community-dwelling elderly. ${ }^{7,9,10)}$ Therefore, we aimed to conduct this study to evaluate the factors associated with dry eye symptoms among elderly Koreans by using data from a nationally representative survey.

\section{METHODS}

\section{Study Population}

This study was based on data acquired from the fifth KNHANES (KNHANES V). Since 1998, the KNHANES has been conducted periodically to assess the health and nutritional status of the civilian and noninstitutionalized population of South Korea. KNHANES V comprises cross-sectional, nationally representative surveys conducted by the Korea Centers for Disease Control and Prevention (KCDC) from 2010 to 2012. Using a complex, stratified, multistage, probability-cluster sampling method, the KCDC selected 31,596 individuals from
11,400 households for possible participation in KNHANES V. Of these individuals, 25,534 agreed to participate, yielding a response rate of $80.8 \%$. Subjects aged $\geq 65$ years were eligible for the study $(n=4,742)$; 557 individuals whose data for dry eye symptoms were unavailable were excluded. This resulted in a total of 4,185 subjects (1,787 men and 2,398 women) who were included in the final statistical analysis. All participants in this survey signed an informed consent form, and the KNHANES was reviewed and approved by the KCDC Institutional Review Board (2010-02CON-21-C, 2011-02CON-06-C, and 2012-01EXP01-2C).

\section{Data Collection and Measurement}

Data were collected during standardized health examinations conducted in specially equipped mobile examination centers. The sequence of administration of the health survey comprised intake, informed consent, anthropometric measurements, blood sampling, and completion of the questionnaire. A standardized questionnaire survey was performed to collect information on age, sex, socioeconomic characteristics, past medical history, current health status and drug use, smoking habits, and other lifestyle-related risk factors.

Marital status was categorized as single or married. The 'single' status included never married, divorced, widowed, or separated from a spouse. Educational level was categorized as $<12$ years of schooling (under high school graduation) or $\geq 12$ years of schooling (equal to or higher than high school graduation). Economic activity status was categorized as employed or unemployed and inactive. Household income levels were divided into quartiles on the basis of inflation-adjusted per capita household income as lowest (1Q), lower middle (2Q), upper middle (3Q), and highest (4Q). The area of residence was categorized as rural or urban on the basis of the Korean administrative districts. Occupation was categorized as indoor or outdoor. People with indoor occupations included managers, professional and related workers, clerks, service and sales workers, housewives, students, and the unemployed. Those with outdoor occupations included people working in agriculture, forestry, fisheries, and crafts; workers operating and assembling equipment and machines; and elementary workers.

To assess the smoking status, the subjects were categorized as current smokers, ex-smokers, or nonsmokers. Nonsmokers were defined as subjects who had smoked $<100$ cigarettes in their lifetime and exsmokers as those who had smoked $\geq 100$ cigarettes in their lifetime but no longer smoked. Monthly alcohol consumption was defined as the consumption of at least one glass of alcohol per month during the previous year. Regular physical activity was evaluated using three categories according to the intensity of exercise. Walking was defined as walking at least 5 times per week for $\geq 30 \mathrm{~min} /$ session. Moderate-intensity physical activity was defined as performing moderate-intensity physical activity at least 5 times per week for $\geq 30 \mathrm{~min} /$ session. Vigorous-intensity physical activity was defined as performing vigorous-intensity physical activity at least 3 times per week for $\geq 20 \mathrm{~min} / \mathrm{session}$. Current duration of exposure to sunlight was categorized as $<2,2-5$, or $\geq 5 \mathrm{~h} / \mathrm{d}$. Sleep duration was self-reported and was assessed by asking, 
Table 1. General characteristics of the study population

\begin{tabular}{|c|c|c|c|}
\hline Characteristic & $\begin{array}{c}\text { No. } \\
\text { (weighted \%) }\end{array}$ & $\begin{array}{l}\text { Standard } \\
\text { error }\end{array}$ & $95 \% \mathrm{Cl}$ \\
\hline \multicolumn{4}{|l|}{ Age (y) } \\
\hline $65-69$ & $1,392(33.0)$ & 0.9 & $31.3-34.8$ \\
\hline $70-74$ & $1,376(29.0)$ & 0.8 & $27.4-30.6$ \\
\hline $75-79$ & $901(23.6)$ & 0.9 & $21.9-25.5$ \\
\hline$\geq 80$ & $516(14.4)$ & 0.8 & $12.9-15.9$ \\
\hline Sex (female) & 2,398 (59.3) & 0.8 & $57.7-60.7$ \\
\hline Marital status (single) & $1,391(36.8)$ & 1.1 & $34.7-38.9$ \\
\hline Educational level (<12 y) & $3,107(82.1)$ & 0.9 & $80.3-83.7$ \\
\hline $\begin{array}{l}\text { Economic activity status } \\
\text { (unemployed and inactive) }\end{array}$ & $2,576(65.3)$ & 1.3 & $62.7-67.8$ \\
\hline \multicolumn{4}{|l|}{ Household income level } \\
\hline Lowest (1Q) & $1,052(26.3)$ & 1.0 & $24.3-28.3$ \\
\hline Lower middle (2Q) & $1,025(26.2)$ & 0.9 & $24.4-28.1$ \\
\hline Upper middle (3Q) & $1,044(25.0)$ & 0.9 & $23.4-26.7$ \\
\hline Highest (4Q) & $991(22.5)$ & 0.9 & $20.7-24.4$ \\
\hline Area of residence (urban) & $2,792(65.1)$ & 2.6 & $59.9-70.0$ \\
\hline Occupation (indoor) & 2,787 (70.5) & 1.3 & $67.8-73.0$ \\
\hline \multicolumn{4}{|l|}{ Smoking status } \\
\hline Nonsmoker & $2,337(60.1)$ & 0.9 & $58.3-61.8$ \\
\hline Ex-smoker & $1,124(27.2)$ & 0.8 & $25.7-28.8$ \\
\hline Current smoker & $490(12.7)$ & 0.7 & $11.5-14.1$ \\
\hline Monthly alcohol consumption & $1,349(33.7)$ & 0.8 & $32.1-35.3$ \\
\hline \multicolumn{4}{|l|}{ Regular physical activity } \\
\hline Walking & $1,486(36.3)$ & 1.1 & $34.2-38.4$ \\
\hline Moderate-intensity & $328(8.1)$ & 0.6 & $7.0-9.4$ \\
\hline Vigorous-intensity & $328(7.5)$ & 0.5 & $6.5-8.6$ \\
\hline \multicolumn{4}{|l|}{ Obesity } \\
\hline General obesity & $1,377(33.1)$ & 1.0 & $31.2-35.0$ \\
\hline Abdominal obesity & $1,517(37.0)$ & 1.1 & $35.0-39.1$ \\
\hline \multicolumn{4}{|c|}{ Duration of exposure to sunlight (h/d) } \\
\hline$<2$ & $2,244(52.3)$ & 1.6 & $49.3-55.4$ \\
\hline $2-5$ & $969(24.1)$ & 1.0 & $22.1-26.2$ \\
\hline$\geq 5$ & $962(23.6)$ & 1.6 & $20.6-26.9$ \\
\hline \multicolumn{4}{|l|}{ Sleep duration (h/d) } \\
\hline$\leq 5$ & $1,148(29.6)$ & 0.9 & $27.8-31.5$ \\
\hline $6-8$ & 2,417 (60.8) & 1.0 & $58.8-62.7$ \\
\hline$\geq 9$ & $377(9.6)$ & 0.7 & $8.4-11.1$ \\
\hline \multicolumn{4}{|l|}{ Mental health status } \\
\hline Perceived stress & $854(22.3)$ & 0.9 & $20.6-24.1$ \\
\hline Depressed mood & $616(16.3)$ & 0.7 & $14.9-17.7$ \\
\hline Suicidal ideation & $832(22.6)$ & 1.0 & $20.7-24.5$ \\
\hline \multicolumn{4}{|l|}{ Medical condition } \\
\hline Hypertension & $2,515(64.2)$ & 1.0 & $62.3-66.0$ \\
\hline Diabetes mellitus & $751(21.7)$ & 0.8 & $20.1-23.4$ \\
\hline Hypercholesterolemia & $752(21.3)$ & 0.9 & $19.7-23.1$ \\
\hline High triglyceride & $529(16.7)$ & 0.8 & $15.2-18.4$ \\
\hline $\begin{array}{l}\text { Low high-density } \\
\text { lipoprotein cholesterol }\end{array}$ & $1,053(29.4)$ & 1.0 & $27.6-31.4$ \\
\hline Anemia & $465(13.9)$ & 0.8 & $12.5-15.4$ \\
\hline \multicolumn{4}{|l|}{ Medical history } \\
\hline Stroke & $208(5.3)$ & 0.5 & $4.5-6.3$ \\
\hline Ischemic heart disease & $279(6.5)$ & 0.5 & $5.6-7.5$ \\
\hline Thyroid disease & $169(4.0)$ & 0.4 & $3.4-4.8$ \\
\hline Rheumatoid arthritis & $148(3.5)$ & 0.3 & $2.9-4.2$ \\
\hline Depressive disorder & $199(4.5)$ & 0.4 & $3.8-5.2$ \\
\hline Cancer & $267(6.8)$ & 0.5 & $5.9-7.8$ \\
\hline
\end{tabular}

Table 1. Continued

\begin{tabular}{lccc}
\hline \multicolumn{1}{c}{ Characteristic } & $\begin{array}{c}\text { No. } \\
\text { (weighted \%) }\end{array}$ & $\begin{array}{c}\text { Standard } \\
\text { error }\end{array}$ & $95 \% \mathrm{Cl}$ \\
\hline $\begin{array}{l}\text { Current medication } \\
\text { Hypertension }\end{array}$ & $2,022(51.7)$ & 1.1 & $49.6-53.8$ \\
$\quad$ Depression & $77(1.7)$ & 0.2 & $1.3-2.3$ \\
Ocular disease & & & \\
Cataract & $1,399(33.6)$ & 1.1 & $31.5-35.9$ \\
Glaucoma & $97(2.3)$ & 0.3 & $1.8-3.1$ \\
Macular degeneration & $20(0.4)$ & 0.1 & $0.2-0.7$ \\
Ophthalmic surgery & $1,329(33.1)$ & 1.0 & $31.1-35.1$ \\
Dry eye symptoms & $806(17.9)$ & 1.0 & $16.0-19.9$ \\
\hline
\end{tabular}

$\mathrm{Cl}$, confidence interval; $\mathrm{Q}$, quartile.

"For how many hours do you usually sleep every day?" The responses were categorized as $\leq 5,6-8$, or $\geq 9 \mathrm{~h} / \mathrm{d}$.

The mental health status was evaluated using perceived stress, depressed mood, and suicidal ideation. Stress perception was measured by asking, "How much stress are you experiencing in your daily life?" The responses were rated on a four-point scale; a 'high' or 'very high' level of stress was considered to indicate perceived stress. Self-reported depressed mood was assessed by asking, "During the past 12 months, did you ever feel so sad or hopeless for 2 weeks or more in a row that you stopped doing usual activities?" Suicidal ideation was assessed by asking, "During the past 12 months, did you ever seriously think about suicide?" The responses to the latter two questions were restricted to 'yes' or 'no.'

Anthropometric measurements, including height, body weight, and waist circumference, were obtained according to standardized guidelines. Body mass index (BMI) was calculated by dividing the body weight by the squared height $\left(\mathrm{kg} / \mathrm{m}^{2}\right)$. General obesity was defined as $\mathrm{BMI} \geq 25.0 \mathrm{~kg} / \mathrm{m}^{2}$. Abdominal obesity was defined as a waist circumference $\geq 90 \mathrm{~cm}$ in a man or $\geq 85 \mathrm{~cm}$ in a woman.

Blood pressure was measured 3 times at 5 -minute intervals by using a standard mercury sphygmomanometer (Baumanometer; WA Baum, Copiague, NY, USA). The average of the second and third measurements was used as the final blood pressure. Fasting plasma glucose (FPG), total cholesterol, triglycerides, and high-density lipoprotein (HDL) cholesterol levels were measured after a fasting period of at least 8-12 hours by using an autoanalyzer (Hitachi Automatic Analyzer 7600; Hitachi, Tokyo, Japan). Hypertension was defined as a systolic blood pressure $\geq 140 \mathrm{~mm} \mathrm{Hg}$, a diastolic blood pressure $\geq 90 \mathrm{~mm} \mathrm{Hg}$, or current use of antihypertensive medication. Diabetes mellitus was defined as an FPG level $\geq 126 \mathrm{mg} / \mathrm{dL}$ or the use of diabetes medication. Hypercholesterolemia was defined as a total cholesterol level $\geq 240$ $\mathrm{mg} / \mathrm{dL}$ or the use of an antidyslipidemic drug. High triglyceride was defined as a triglyceride level $\geq 200 \mathrm{mg} / \mathrm{dL}$ after fasting for at least 12 hours. Low HDL cholesterol was defined as an HDL cholesterol level $<40 \mathrm{mg} / \mathrm{dL}$ after fasting for at least 8 hours. Anemia was defined as a hemoglobin level $<13 \mathrm{~g} / \mathrm{dL}$ in a man or $<12 \mathrm{~g} / \mathrm{dL}$ in a woman.

Medical histories of stroke, ischemic heart disease, thyroid disease, rheumatoid arthritis, depression, and cancer were assessed using 
Table 2. Comparison between participants with and without dry eye symptoms

\begin{tabular}{|c|c|c|c|c|c|}
\hline \multirow{2}{*}{ Variable } & \multicolumn{2}{|c|}{ With dry eye symptoms ( $\mathrm{n}=806)$} & \multicolumn{2}{|c|}{ Without dry eye symptoms $(n=3,379)$} & \multirow{2}{*}{ P-value } \\
\hline & No. (weighted \%) & SE & No. (weighted \%) & SE & \\
\hline Age (y) & & & & & $<0.001$ \\
\hline $65-69$ & $287(36.9)$ & 2.2 & $1,105(32.2)$ & 1.0 & \\
\hline $70-74$ & $266(28.8)$ & 1.9 & $1,110(29.0)$ & 0.9 & \\
\hline $75-79$ & $163(22.8)$ & 1.8 & $738(23.8)$ & 1.0 & \\
\hline$\geq 80$ & $90(11.4)$ & 1.7 & $426(15.0)$ & 0.8 & \\
\hline Sex (female) & $563(71.2)$ & 1.9 & $1,835(56.7)$ & 0.9 & $<0.001$ \\
\hline Marital status (single) & $302(40.4)$ & 2.3 & $1,089(36.0)$ & 1.1 & 0.063 \\
\hline Educational level $(<12 \mathrm{y})$ & $604(81.1)$ & 1.8 & $2,503(82.3)$ & 0.9 & 0.550 \\
\hline Economic activity status (unemployed and inactive) & $561(71.4)$ & 2.1 & $2,015(63.9)$ & 1.5 & 0.003 \\
\hline Household income level & & & & & 0.377 \\
\hline Lowest (1Q) & $188(24.0)$ & 2.1 & $864(26.8)$ & 1.1 & \\
\hline Lower middle (2Q) & $200(27.2)$ & 2.0 & $825(26.0)$ & 1.1 & \\
\hline Upper middle (3Q) & $204(27.6)$ & 2.2 & $840(24.5)$ & 0.9 & \\
\hline Highest (4Q) & $200(21.2)$ & 1.9 & $791(22.8)$ & 1.0 & \\
\hline Area of residence (urban) & $588(73.7)$ & 3.2 & $2,204(63.2)$ & 2.7 & 0.001 \\
\hline Occupation (indoor) & $607(78.0)$ & 2.0 & $2,180(68.8)$ & 1.5 & $<0.001$ \\
\hline Smoking status & & & & & $<0.001$ \\
\hline Nonsmoker & $543(70.8)$ & 2.0 & $1,794(57.7)$ & 1.0 & \\
\hline Ex-smoker & $168(19.6)$ & 1.7 & $956(28.9)$ & 0.9 & \\
\hline Current smoker & $64(9.6)$ & 1.4 & $426(13.4)$ & 0.7 & \\
\hline Monthly alcohol consumption & $225(28.8)$ & 1.9 & $1,124(34.8)$ & 0.9 & 0.007 \\
\hline \multicolumn{6}{|l|}{ Regular physical activity } \\
\hline Walking & $269(33.5)$ & 2.1 & $1,217(36.9)$ & 1.2 & 0.151 \\
\hline Moderate-intensity & $53(7.3)$ & 1.3 & $275(8.3)$ & 0.7 & 0.466 \\
\hline Vigorous-intensity & $70(8.5)$ & 1.2 & $258(7.3)$ & 0.6 & 0.317 \\
\hline \multicolumn{6}{|l|}{ Obesity } \\
\hline General obesity & $284(37.9)$ & 2.1 & $1,093(32.0)$ & 1.1 & 0.011 \\
\hline Abdominal obesity & $308(39.8)$ & 2.2 & $1,209(36.4)$ & 1.1 & 0.130 \\
\hline Duration of exposure to sunlight (h/d) & & & & & 0.305 \\
\hline$<2$ & $468(55.0)$ & 2.7 & $1,776(51.8)$ & 1.7 & \\
\hline $2-5$ & $173(24.3)$ & 2.1 & $796(24.0)$ & 1.2 & \\
\hline$\geq 5$ & $164(20.8)$ & 2.1 & $798(24.2)$ & 1.7 & \\
\hline Sleep duration (h/d) & & & & & 0.002 \\
\hline$\leq 5$ & $255(33.1)$ & 2.0 & $893(28.8)$ & 1.0 & \\
\hline $6-8$ & $463(60.9)$ & 2.0 & $1,954(60.7)$ & 1.1 & \\
\hline$\geq 9$ & $55(6.0)$ & 1.0 & $322(10.4)$ & 0.8 & \\
\hline \multicolumn{6}{|l|}{ Mental health status } \\
\hline Perceived stress & $201(28.1)$ & 2.1 & $653(21.0)$ & 1.0 & 0.001 \\
\hline Depressed mood & $148(19.4)$ & 1.6 & $468(15.6)$ & 0.8 & 0.020 \\
\hline Suicidal ideation & $200(28.0)$ & 2.2 & $632(21.4)$ & 1.0 & 0.003 \\
\hline \multicolumn{6}{|l|}{ Medical condition } \\
\hline Hypertension & $499(64.0)$ & 2.1 & $2,016(64.2)$ & 1.1 & 0.933 \\
\hline Diabetes mellitus & $155(22.6)$ & 2.2 & $596(21.5)$ & 0.9 & 0.643 \\
\hline Hypercholesterolemia & $189(28.3)$ & 2.1 & $563(19.8)$ & 0.9 & $<0.001$ \\
\hline High triglyceride & $108(17.4)$ & 1.8 & $421(16.6)$ & 0.9 & 0.666 \\
\hline Low high-density lipoprotein cholesterol & $175(25.5)$ & 2.0 & $878(30.3)$ & 1.1 & 0.034 \\
\hline Anemia & $89(14.5)$ & 1.7 & $376(13.8)$ & 0.8 & 0.678 \\
\hline \multicolumn{6}{|l|}{ Medical history } \\
\hline Stroke & $36(4.5)$ & 0.9 & $172(5.5)$ & 0.5 & 0.341 \\
\hline Ischemic heart disease & $59(7.5)$ & 1.3 & $220(6.3)$ & 0.5 & 0.390 \\
\hline Thyroid disease & $53(6.1)$ & 1.0 & $116(3.6)$ & 0.4 & 0.004 \\
\hline Rheumatoid arthritis & $28(2.9)$ & 0.6 & $120(3.6)$ & 0.4 & 0.327 \\
\hline Depressive disorder & $56(5.9)$ & 1.0 & $143(4.1)$ & 0.4 & 0.052 \\
\hline Cancer & $47(5.3)$ & 0.9 & $220(7.1)$ & 0.5 & 0.107 \\
\hline
\end{tabular}


Table 2. Continued

\begin{tabular}{|c|c|c|c|c|c|}
\hline \multirow{2}{*}{ Variable } & \multicolumn{2}{|c|}{ With dry eye symptoms $(n=806)$} & \multicolumn{2}{|c|}{ Without dry eye symptoms $(n=3,379)$} & \multirow{2}{*}{ P-value } \\
\hline & No. (weighted \%) & SE & No. (weighted \%) & SE & \\
\hline \multicolumn{6}{|l|}{ Current medication } \\
\hline Hypertension & $434(55.6)$ & 2.2 & $1,588(50.8)$ & 1.2 & 0.054 \\
\hline Depression & $22(2.4)$ & 0.6 & $55(1.6)$ & 0.3 & 0.196 \\
\hline \multicolumn{6}{|l|}{ Ocular disease } \\
\hline Cataract & $368(48.1)$ & 2.3 & $1,031(30.6)$ & 1.2 & $<0.001$ \\
\hline Glaucoma & $33(3.9)$ & 0.8 & $64(2.0)$ & 0.3 & $<0.001$ \\
\hline Macular degeneration & $9(1.1)$ & 0.5 & $11(0.3)$ & 0.1 & $<0.001$ \\
\hline Ophthalmic surgery & $322(44.2)$ & 2.3 & $1,007(30.7)$ & 1.1 & $<0.001$ \\
\hline
\end{tabular}

P-values were determined using the chi-square test.

SE, standard error; Q, quartile.

questions asking whether the subjects had received a diagnosis of these diseases from a doctor. Current medication was investigated for hypertension and depression.

\section{Definition of Dry Eye Symptoms and Ocular Diseases}

To assess the prevalence of dry eye symptoms, only the questionnaire on dry eye symptoms was used, and the subjects were asked the question, "Until now, have you ever had a symptom of dry eye before, for example, a sense of irritation or dryness of the eyes?" To this question, if the subjects responded as having experienced dry eye symptoms 'persistently', they were categorized as having dry eye symptoms. However, if the subjects had experienced a symptom 'sometimes' or 'occasionally' or had never experienced a symptom, they were categorized as not having dry eye symptoms. Other ophthalmologic questionnaires included questions on the history of ophthalmic surgery and history of diagnoses by an ophthalmologist, including cataract, glaucoma, and age-related macular degeneration.

\section{Statistical Analyses}

All estimates were calculated according to sample weights, which were evaluated by considering the sampling rate, response rate, and age and sex proportions of the reference population. The analysis was adjusted for the complex sample design of the survey. Categorical data were expressed as frequencies and standard errors or $95 \%$ confidence intervals (CIs). The comparison between those with and those without dry eye symptoms was performed using the chi-square test for categorical data. Logistic regression analyses were used to analyze the relationship between dry eye symptoms and related factors. All tests were two-sided, and P-values $<0.05$ were considered to indicate statistical significance. The statistical analyses were performed using IBM SPSS Statistics for Windows/Macintosh ver. 24.0. (IBM Corp., Armonk, NY, USA).

\section{RESULTS}

Table 1 summarizes the general characteristics of the study population. The overall prevalence of dry eye symptoms was $17.9 \%$ (95\% CI, $16.0-19.9)$; 1,399 participants (33.6\%) had a history of cataract, and
1,329 (33.1\%) had a history of ophthalmic surgery.

Table 2 compares diverse basic variables between participants with and without dry eye symptoms. We found that the older the age, the less prevalent the dry eye symptoms was. The following factors were significantly associated with dry eye symptoms: age, female sex, unemployed and inactive status, urban residence, indoor occupation, smoking status, monthly alcohol consumption, general obesity, sleep duration, perceived stress, depressed mood, suicidal ideation, hypercholesterolemia, low HDL cholesterol, a history of thyroid disease, a history of ophthalmic surgery, and a history of cataract, glaucoma, or age-related macular degeneration (all P-values $<0.05$ ).

Table 3 shows the logistic regression analysis of associations between dry eye symptoms and clinical variables.

Table 4 shows the results for the final model of multivariate analysis, with adjustment for all variables that were statistically significant in the univariate analysis. This final, fully adjusted analysis showed that female sex (adjusted odds ratio [aOR], 1.806; 95\% CI, 1.410-2.313), a history of cataract (aOR, 1.683; 95\% CI, 1.255-2.255), suicidal ideation (aOR, 1.414; 95\% CI, 1.070-1.870), hypercholesterolemia (aOR, 1.289; 95\% CI, 1.025-1.621), age $\geq 80$ years (aOR, 0.538; 95\% CI, 0.337-0.859), and sleep duration $\geq 9 \mathrm{~h} / \mathrm{d}$ (aOR, 0.524; 95\% CI, 0.330-0.834) were significantly associated with dry eye symptoms.

\section{DISCUSSION}

The aim of this study was to evaluate the factors associated with dry eye symptoms in Koreans aged $\geq 65$ years. Knowledge about the factors associated with dry eye disease is very important because it can help physicians understand the pathophysiology and choose the methods of treatment or prevention for elderly patients with dry eye symptoms. Previous studies have indicated the following as well-known risk factors for dry eye disease: older age, female sex, pregnancy, oral contraceptive use, menopausal status, postmenopausal estrogen therapy, androgen deficiency, diabetes mellitus, alcohol, smoking, caffeine, low-humidity environment, refractory surgery, and medications such as antihistamine, tricyclic antidepressants, selective serotonin reuptake inhibitors, diuretics, beta-blockers, anti-cholinergics including anxiolytics, and antipsychotics. ${ }^{11)}$ In addition, recent studies using the 
Table 3. Logistic regression analysis of the association between dry eye symptoms and clinical variables before and after adjustment for age and sex

\begin{tabular}{|c|c|c|}
\hline Variable & Crude & Age- and sex-adjusted \\
\hline \multicolumn{3}{|l|}{ Age (y) } \\
\hline $65-69$ & Reference & Reference \\
\hline $70-74$ & 0.865 (0.689-1.085) & $0.852(0.676-1.074)$ \\
\hline $75-79$ & $0.835(0.653-1.067)$ & $0.787(0.614-1.008)$ \\
\hline$\geq 80$ & $0.664(0.462-0.956)$ & $0.604(0.416-0.875)$ \\
\hline Sex (female) & $1.890(1.537-2.324)$ & $1.955(1.587-2.408)$ \\
\hline Marital status (single) & $1.209(0.989-1.479)$ & $1.005(0.799-1.265)$ \\
\hline Educational level $(<12 \mathrm{y})$ & $0.928(0.724-1.188)$ & $0.693(0.525-0.914)$ \\
\hline $\begin{array}{l}\text { Economic activity status } \\
\text { (unemployed and inactive) }\end{array}$ & $1.411(1.127-1.768)$ & $1.422(1.124-1.798)$ \\
\hline \multicolumn{3}{|l|}{ Household income level } \\
\hline Lowest (1Q) & Reference & Reference \\
\hline Lower middle (2Q) & $1.162(0.886-1.523)$ & $1.147(0.874-1.506)$ \\
\hline Upper middle (3Q) & $1.257(0.928-1.703)$ & $1.256(0.924-1.708)$ \\
\hline Highest (4Q) & $1.040(0.769-1.406)$ & $1.042(0.768-1.412)$ \\
\hline Area of residence (urban) & $1.626(1.213-2.179)$ & $1.612(1.200-2.165)$ \\
\hline Occupation (indoor) & 1.601 (1.255-2.042) & 1.585 (1.232-2.039) \\
\hline \multicolumn{3}{|l|}{ Smoking status } \\
\hline Nonsmoker & Reference & Reference \\
\hline Ex-smoker & $0.553(0.435-0.703)$ & $0.804(0.566-1.143)$ \\
\hline Current smoker & $0.585(0.418-0.819)$ & $0.819(0.559-1.200)$ \\
\hline Monthly alcohol consumption & $0.756(0.617-0.926)$ & $0.941(0.757-1.168)$ \\
\hline \multicolumn{3}{|l|}{ Regular physical activity } \\
\hline Walking & $0.862(0.704-1.056)$ & $0.905(0.735-1.115)$ \\
\hline Moderate-intensity & $0.864(0.582-1.281)$ & $0.847(0.563-1.275)$ \\
\hline Vigorous-intensity & $.188(0.847-1.664)$ & $1.261(0.897-1.773)$ \\
\hline \multicolumn{3}{|l|}{ Obesity } \\
\hline General obesity & $1.292(1.061-1.574)$ & $1.157(0.949-1.411)$ \\
\hline Abdominal obesity & $1.157(0.958-1.396)$ & $1.036(0.851-1.263)$ \\
\hline \multicolumn{3}{|l|}{$\begin{array}{l}\text { Duration of exposure to } \\
\text { sunlight }(\mathrm{h} / \mathrm{d})\end{array}$} \\
\hline$<2$ & Reference & Reference \\
\hline $2-5$ & $0.951(0.719-1.257)$ & $0.999(0.753-1.326)$ \\
\hline$\geq 5$ & $0.806(0.617-1.053)$ & $0.879(0.672-1.151)$ \\
\hline \multicolumn{3}{|l|}{ Sleep duration (h/d) } \\
\hline$\leq 5$ & Reference & \\
\hline $6-8$ & $0.875(0.726-1.054)$ & $0.929(0.766-1.126)$ \\
\hline$\geq 9$ & $0.502(0.338-0.746)$ & $0.550(0.370-0.816)$ \\
\hline \multicolumn{3}{|l|}{ Mental health status } \\
\hline Perceived stress & $1.470(1.167-1.851)$ & $1.298(1.033-1.630)$ \\
\hline Depressed mood & $1.305(1.043-1.633)$ & $1.211(0.968-1.516)$ \\
\hline Suicidal ideation & $1.435(1.134-1.817)$ & $1.371(1.091-1.725)$ \\
\hline \multicolumn{3}{|l|}{ Medical condition } \\
\hline Hypertension & $0.992(0.812-1.211)$ & $0.941(0.772-1.147)$ \\
\hline Diabetes mellitus & $1.066(0.813-1.398)$ & $1.078(0.823-1.413)$ \\
\hline Hypercholesterolemia & $1.604(1.267-2.031)$ & $1.416(1.122-1.788)$ \\
\hline High triglyceride & $1.062(0.808-1.397)$ & $1.021(0.774-1.346)$ \\
\hline $\begin{array}{l}\text { Low high-density } \\
\text { lipoprotein cholesterol }\end{array}$ & 0.786 (0.629-0.982) & $0.859(0.687-1.075)$ \\
\hline Anemia & $1.066(0.787-1.443)$ & $1.080(0.797-1.464)$ \\
\hline \multicolumn{3}{|l|}{ Medical history } \\
\hline Stroke & $0.812(0.528-1.248)$ & $0.912(0.592-1.407)$ \\
\hline Ischemic heart disease & $1.201(0.790-1.828)$ & $1.259(0.823-1.928)$ \\
\hline Thyroid disease & $1.741(1.184-2.560)$ & $1.435(0.964-2.136)$ \\
\hline Rheumatoid arthritis & $0.789(0.491-1.269)$ & $0.664(0.410-1.077)$ \\
\hline Depressive disorder & $1.469(0.994-2.173)$ & $1.229(0.822-1.836)$ \\
\hline Cancer & $0.730(0.497-1.073)$ & $0.743(0.500-1.105)$ \\
\hline
\end{tabular}

(Continued to the next page)
Table 3. Continued

\begin{tabular}{lll}
\hline \multicolumn{1}{c}{ Variable } & \multicolumn{1}{c}{ Crude } & Age- and sex-adjusted \\
\hline $\begin{array}{c}\text { Current medication } \\
\text { Hypertension }\end{array}$ & $1.212(0.997-1.473)$ & $1.158(0.952-1.409)$ \\
$\quad$ Depression & $1.471(0.816-2.651)$ & $1.241(0.686-2.246)$ \\
Ocular disease & & \\
Cataract & $2.107(1.711-2.595)$ & $2.199(1.781-2.716)$ \\
Glaucoma & $1.994(1.183-3.363)$ & $2.123(1.208-3.730)$ \\
Macular degeneration & $4.294(1.501-12.279)$ & $4.867(1.535-15.434)$ \\
Ophthalmic surgery & $1.788(1.441-2.218)$ & $1.802(1.447-2.244)$ \\
\hline
\end{tabular}

Values are presented as odds ratio (95\% confidence interval). Q, quartile.

KNHANES in South Korea suggested the following factors were associated with dry eye disease: dyslipidemia, degenerative arthritis, rheumatoid arthritis, thyroid disease, renal failure, stress, depression, history of ocular surgery, urban residence, indoor occupation, low education level, regular exercise, sleep duration, inadequate sun exposure, and low 25-hydroxyvitamin D levels. ${ }^{6,12-14)}$ Therefore, we selected multiple variables that had been suggested as relevant factors for dry eye disease and evaluated the relationships between these variables and dry eye symptoms in Koreans aged $\geq 65$ years.

We found that the prevalence of dry eye symptoms was $17.9 \%$ and that the factors associated with dry eye symptoms were female sex, a history of cataract, suicidal ideation, and hypercholesterolemia. The prevalence of dry eye symptoms in our study was lower than that in studies conducted among the community-dwelling elderly population in South Korea. A study of people aged $\geq 65$ years in the Yongin area using a six-item questionnaire to assess dry eye symptoms found a crude prevalence of $30.3 \% .^{7)}$ Another community-dwelling populationbased study of people aged $\geq 50$ years in the Incheon area found the prevalence of dry eye symptoms to be $26.2 \%{ }^{9}{ }^{9}$ That study used a selfadministered six-item questionnaire similar to the one used in the Yongin study. Several studies from other countries have also reported a high prevalence of dry eye disease. ${ }^{5,15)}$ The variation in the prevalence could be attributed to differences in race, ethnicity, and age of the study populations; the lack of a standardized diagnostic definition; and the diversity of study methods.

Although some previous studies found no association between aging and the prevalence of dry eye disease, ${ }^{5,9,15,16)}$ other studies showed that the prevalence of dry eye disease increases with age. ${ }^{6,17)}$ However, our study showed that the prevalence and OR of dry eye symptoms tended to decrease with age. To date, no study has reported that old age leads to a less prevalence of dry eye symptoms. Our result can be explained as follows. First, the proportion of the group aged $\geq 80$ years in the study population was $14.4 \%(n=516)$, and it was much lower than that of the other age groups. This low participation rate may have affected our results regarding the prevalence of dry eye symptoms in the group aged $\geq 80$ years. Second, subjects older than 80 years who actually have dry eye symptoms may not have been selected as participants in the KNHANES. Third, the elderly people presumably do not use electronic appliances, such as computers and cellular phones, as 
Table 4. Multivariate adjusted evaluation of the factors associated with dry eye symptoms

\begin{tabular}{lcr}
\multicolumn{1}{c}{ Variable } & Adjusted OR (95\% Cl) & P-value \\
\hline Age (y) & Reference & \\
$65-69$ & $0.801(0.614-1.045)$ & 0.102 \\
$70-74$ & $0.753(0.551-1.028)$ & 0.074 \\
$75-79$ & $0.538(0.337-0.859)$ & 0.009 \\
$\geq 80$ & $1.806(1.410-2.313)$ & $<0.001$ \\
Sex (female) & $0.803(0.588-1.096)$ & 0.166 \\
Educational level (<12 y) & $1.093(0.682-1.753)$ & 0.711 \\
Economic activity status & & \\
$\quad$ (unemployed and inactive) & $1.358(0.988-1.868)$ & 0.060 \\
Area of residence (urban) & $1.114(0.685-1.813)$ & 0.662 \\
Occupation (indoor) & & \\
Sleep duration (h/d) & Reference & 0.726 \\
$\quad \leq 5$ & $0.961(0.771-1.199)$ & 0.006 \\
$\quad 6-8$ & $0.524(0.330-0.834)$ & 0.316 \\
$\quad \geq 9$ & $1.153(0.873-1.522)$ & 0.015 \\
Perceived stress & $1.414(1.070-1.870)$ & 0.030 \\
Suicidal ideation & $1.289(1.025-1.621)$ & 0.001 \\
Hypercholesterolemia & $1.683(1.255-2.255)$ & 0.186 \\
Cataract & $1.533(0.813-2.889)$ & \\
Glaucoma & $2.890(0.928-8.999)$ & 0.067 \\
Macular degeneration & $1.294(0.952-1.757)$ & 0.099 \\
Ophthalmic surgery & & \\
\hline
\end{tabular}

Adjusted ORs and 95\% Cls were estimated using the multiple logistic regression analysis with adjustment for all variables. All variables were statistically significant in the univariate analysis.

$\mathrm{OR}$, odds ratio; $\mathrm{Cl}$, confidence interval.

much as the young adult population does, thereby resulting in less eye fatigue and dry eye symptoms in the elderly. Finally, the sensitivity of the cornea may possibly be reduced in the elderly people; hence, they are less likely to report dry eye symptoms.

Some studies have shown that the prevalence of dry eye disease is higher in women, ${ }^{6,7,17)}$ but other studies have found no difference between the sexes. ${ }^{5,16)}$ Our study showed that dry eye symptoms were more prevalent in women than in men and that female sex was significantly associated with dry eye symptoms. The higher prevalence in women may be attributable to the hormonal or metabolic changes associated with menopause, post-menopausal hormone replacement, androgen deficiency, or use of oral contraceptives. ${ }^{11,18)}$ One study showed that women in their 60s had less tear production than did similarly aged men, ${ }^{19)}$ and another study suggested that sex hormones may have an important effect on ocular surface conditions owing to their effects on lacrimal glands, meibomian glands, conjunctival goblet cell density, and ocular surface sensitivity. ${ }^{20)}$ Because the participants in our study were postmenopausal, hormones may have affected the manifestation of dry eye. However, we did not investigate hormonal factors in this study.

Notably, suicidal ideation was significantly associated with dry eye symptoms in our study. Although one study showed an association between depression and dry eye disease in the community-dwelling elderly population in South Korea, ${ }^{10)}$ no study reported the relationship between suicidal ideation and dry eye symptoms in the elderly.
One study reported that proinflammatory cytokines such as interleukin-1, interleukin-6, and tumor necrosis factor- $\alpha$ can cause inflammation of the ocular surface in dry eye disease, affecting neurotransmission and producing or enhancing a negative mood. ${ }^{21)}$ Considering the high suicide rate in South Korea, our study population may have included many elderly people with suicidal ideation. In addition, female sex, aging, and hormonal effects are common risk factors for dry eye disease and depression. ${ }^{7,15)}$ However, our study did not calibrate the effects of hormones. Moreover, because a person with suicidal ideation may have other psychiatric problems, such as stress, depression, and insomnia, dry eye symptoms could be related to psychiatric medications such as antidepressants and anxiolytics.

The present study found that hypercholesterolemia was also significantly associated with dry eye symptoms. One study suggested that hypercholesterolemia is associated with meibomian gland dysfunction, which is a primary cause of evaporative dry eye disease. ${ }^{22)}$ This effect could be explained as a result of an increase in cholesterol in the meibomian gland, which may raise its lipid melting point from the normal value of $30^{\circ} \mathrm{C}-34^{\circ} \mathrm{C}$ to $46^{\circ} \mathrm{C}$, leading to increased viscosity that may cause plugging of the meibomian orifice and aggravation of meibomian gland disease. ${ }^{22)}$ Considering the high prevalence of dyslipidemia worldwide, further studies are needed to confirm the association between dyslipidemia and dry eye symptoms.

Remarkably, we found that a history of cataract was a significantly relevant factor for dry eye symptoms. Although one study showed that cataract surgery can cause dry eye, ${ }^{23)}$ no study found an association between cataract itself and dry eye symptoms. In South Korea, the prevalence of cataract was $42.28 \%$ in the population aged $\geq 40$ years and $94.15 \%$ in those aged $\geq 70$ years. In addition, the prevalence of previous cataract surgery was $7.75 \%$ in the population aged $\geq 40$ years and $30.63 \%$ in that aged $\geq 70$ years. ${ }^{24)}$ Therefore, many subjects who had cataract or who had already undergone cataract surgery may have been included in our study. There are many known risk factors for cataract, such as aging, female sex, smoking, diabetes, inflammation of the eye, glaucoma and related treatment, ultraviolet irradiation, steroids, and hormone replacement therapy, ${ }^{25)}$ and some of these factors overlap with the risk factors for dry eye disease. Therefore, the inference of a link between a history of cataract and dry eye symptoms could be incorrectly reached because of hasty generalization. Moreover, further studies are needed to clarify the association between cataract and dry eye symptoms.

Our study found that sleep duration $\geq 9 \mathrm{~h} / \mathrm{d}$ was significantly associated with dry eye symptoms. Similarly, one Korean study on the adult population aged $\geq 20$ years old reported that 'mild short' $(5 \mathrm{~h} / \mathrm{d})$ or 'severe short' $(\leq 4 \mathrm{~h} / \mathrm{d})$ sleepers were at a significantly higher risk for dry eye symptoms. ${ }^{12)}$ Another study suggested that rapid eye movement during sleep serves not only to increase lacrimal secretion but also to humidify and lubricate the ocular surface. ${ }^{26)}$ In another experimental study, sleep deprivation caused the induction of tear hyperosmolarity, shortening of tear film break-up time, and reduction of tear secretion, all of which can lead to ocular surface disease. ${ }^{27)}$ In this study, however, 
sleep duration was self-reported and may have been difficult to recall accurately, resulting in possible underestimation or overestimation of sleep duration.

Our study has several potential limitations. First, because it was a cross-sectional study, the cause-and-effect relationships between dry eye symptoms and associated factors were unclear. Second, we used a self-administered questionnaire for evaluating dry eye symptoms without performing objective ophthalmic examinations. Some previous studies used objective measures, such as the Schirmer test, rose Bengal stain, and tear film break-up time, for diagnosing dry eye disease, ${ }^{17,27)}$ but these objective tests had low association with dry eye symptoms and poor reproducibility. ${ }^{11,28)}$ No standard tests are available for assessing dry eye disease. As a result, enquiring about the associated symptoms of dry eye is one of the most reliable diagnostic techniques, ${ }^{12)}$ and various questionnaires with different sensitivities and specificities, such as the Ocular Surface Disease Index Questionnaire, the National Eye Institute Visual Function Questionnaire, the McMonnies Dry Eye Questionnaire (DEQ), and 12-item Short-Form Health Status Questionnaire, have been used for the symptomatic assessment of dry eye. ${ }^{11,23)}$ Of these dry eye symptoms questionnaires, McMonnies DEQ has been widely regarded as a standard questionnaire for screening dry eye disease, with reported sensitivity ranging from $87 \%$ to $98 \%$ and specificity ranging of from $87 \%$ to $97 \%{ }^{4,29)}$ In addition, a recent study reported that the Standard Patient Evaluation of Eye Dryness Questionnaire is useful for the epidemiologic study of dry eye disease because of its high sensitivity and specificity. ${ }^{30)}$ However, self-reporting of dry eye symptoms by using the questionnaire in our study may not accurately reflect the dry eye condition because the pain sensitivity of elderly people is different. Moreover, the question regarding dry eye symptoms was not specific to dry eye disease, and it would be difficult to distinguish dry eye disease from ocular surface diseases, such as meibomian gland disease, allergic conjunctivitis, and chronic infectious conjunctivitis. Third, most elderly people may have had many other important systemic diseases; therefore, they may have neglected or lacked interest in their dry eye symptoms. As a result, the prevalence of dry eye symptoms may be underestimated. Finally, the possibility of recall bias in the KNHANES data should be considered because many variables were self-reported.

Despite these limitations, our study has some significant advantages. To the best of our knowledge, this is the first large population-based study to investigate the factors associated with dry eye symptoms in elderly Koreans in South Korea. This study was based on a recent survey of a nationwide, population-based, representative sample of Koreans, and all analyses in this study were completely based on sample weights and adjusted for the complex sample design of the survey. Therefore, these results can be generalized to the Korean elderly population.

In conclusion, this study has demonstrated that female sex, a history of cataract, suicidal ideation, and hypercholesterolemia may be the risk factors for dry eye symptoms, whereas sleep duration $\geq 9 \mathrm{~h} / \mathrm{d}$ can be a protective factor against dry eye symptoms in elderly Koreans.
The results of this study suggest that physicians should pay more attention to dry eye symptoms in the elderly patients in order to improve their quality of life. Given the prevalence of dry eye symptoms in the Korean elderly population, further studies are required to examine the causal relationships between dry eye symptoms and associated factors by using diagnostic tools with much higher sensitivity and specificity.

\section{CONFLICT OF INTEREST}

No potential conflict of interest relevant to this article was reported.

\section{REFERENCES}

1. Statistics Korea. Population projections for Korea: 2015-2065 (based on the 2015 population census). Daejeon: Statistics Korea; 2017.

2. Joo CK. Ocular disease in the elderly. J Korean Med Assoc 2005;48:22635.

3. The definition and classification of dry eye disease: report of the Definition and Classification Subcommittee of the International Dry Eye WorkShop (2007). Ocul Surf 2007;5:75-92.

4. Tan LL, Morgan P, Cai ZQ, Straughan RA. Prevalence of and risk factors for symptomatic dry eye disease in Singapore. Clin Exp Optom 2015;98:45-53.

5. Uchino M, Dogru M, Yagi Y, Goto E, Tomita M, Kon T, et al. The features of dry eye disease in a Japanese elderly population. Optom Vis Sci 2006;83:797-802.

6. Ahn JM, Lee SH, Rim TH, Park RJ, Yang HS, Kim TI, et al. Prevalence of and risk factors associated with dry eye: the Korea National Health and Nutrition Examination Survey 2010-2011. Am J Ophthalmol 2014;158:1205-14.

7. Han SB, Hyon JY, Woo SJ, Lee JJ, Kim TH, Kim KW. Prevalence of dry eye disease in an elderly Korean population. Arch Ophthalmol 2011;129:633-8.

8. Miljanovic B, Dana R, Sullivan DA, Schaumberg DA. Impact of dry eye syndrome on vision-related quality of life. Am J Ophthalmol 2007;143: 409-15.

9. Jeong HS, Lim JS, Oh DK, Chi MJ, Paik HJ, Shyn KH, et al. Prevalence and risk factors of dry eye syndrome in the Incheon area. J Korean Ophthalmol Soc 2011;52:1135-41.

10. Kim KW, Han SB, Han ER, Woo SJ, Lee JJ, Yoon JC, et al. Association between depression and dry eye disease in an elderly population. Invest Ophthalmol Vis Sci 2011;52:7954-8.

11. The epidemiology of dry eye disease: report of the Epidemiology Subcommittee of the International Dry Eye WorkShop (2007). Ocul Surf 2007;5:93-107.

12. Lee W, Lim SS, Won JU, Roh J, Lee JH, Seok H, et al. The association between sleep duration and dry eye syndrome among Korean adults. Sleep Med 2015;16:1327-31.

13. Roh HC, Lee JK, Kim M, Oh JH, Chang MW, Chuck RS, et al. Systemic comorbidities of dry eye syndrome: the Korean National Health and Nutrition Examination Survey V, 2010 to 2012. Cornea 2016;35:187-92.

14. Yoon SY, Bae SH, Shin YJ, Park SG, Hwang SH, Hyon JY, et al. Low serum 25-hydroxyvitamin D levels are associated with dry eye syndrome. PLoS One 2016;11:e0147847. 
15. Lin PY, Tsai SY, Cheng CY, Liu JH, Chou P, Hsu WM. Prevalence of dry eye among an elderly Chinese population in Taiwan: the Shihpai Eye Study. Ophthalmology 2003;110:1096-101.

16. Schein OD, Munoz B, Tielsch JM, Bandeen-Roche K, West S. Prevalence of dry eye among the elderly. Am J Ophthalmol 1997;124:723-8.

17. Schaumberg DA, Sullivan DA, Buring JE, Dana MR. Prevalence of dry eye syndrome among US women. Am J Ophthalmol 2003;136:318-26.

18. Schaumberg DA, Buring JE, Sullivan DA, Dana MR. Hormone replacement therapy and dry eye syndrome. JAMA 2001;286:2114-9.

19. Mathers WD, Stovall D, Lane JA, Zimmerman MB, Johnson S. Menopause and tear function: the influence of prolactin and sex hormones on human tear production. Cornea 1998;17:353-8.

20. Krenzer KL, Dana MR, Ullman MD, Cermak JM, Tolls DB, Evans JE, et al. Effect of androgen deficiency on the human meibomian gland and ocular surface. J Clin Endocrinol Metab 2000;85:4874-82.

21. Raison CL, Capuron L, Miller AH. Cytokines sing the blues: inflammation and the pathogenesis of depression. Trends Immunol 2006;27:2431.

22. Pinna A, Blasetti F, Zinellu A, Carru C, Solinas G. Meibomian gland dysfunction and hypercholesterolemia. Ophthalmology 2013;120: 2385-9.
23. Kasetsuwan N, Satitpitakul V, Changul T, Jariyakosol S. Incidence and pattern of dry eye after cataract surgery. PLoS One 2013;8:e78657.

24. Park SJ, Lee JH, Kang SW, Hyon JY, Park KH. Cataract and cataract surgery: nationwide prevalence and clinical determinants. J Korean Med Sci 2016;31:963-71.

25. Abraham AG, Condon NG, West Gower E. The new epidemiology of cataract. Ophthalmol Clin North Am 2006;19:415-25.

26. Murube J. REM sleep: tear secretion and dreams. Ocul Surf 2008;6:2-8.

27. Lee YB, Koh JW, Hyon JY, Wee WR, Kim JJ, Shin YJ. Sleep deprivation reduces tear secretion and impairs the tear film. Invest Ophthalmol Vis Sci 2014;55:3525-31.

28. Nichols KK, Nichols JJ, Mitchell GL. The lack of association between signs and symptoms in patients with dry eye disease. Cornea 2004;23: 762-70.

29. Erickson PM, Stapleton F, Giannakopoulos E, Erickson DB, Sweeney D. Reliability of the McMonnies dry eye questionnaire. Invest Ophthalmol Vis Sci 2002;43:3068.

30. Asiedu K, Kyei S, Mensah SN, Ocansey S, Abu LS, Kyere EA. Ocular Surface Disease Index (OSDI) versus the Standard Patient Evaluation of Eye Dryness (SPEED): a study of a nonclinical sample. Cornea 2016;35:175-80. 